\title{
Boundary effects on flux penetration in disordered superconductors
}

\author{
André A. Moreira, ${ }^{1}$ José S. Andrade, Jr., ${ }^{1}$ Josué Mendes Filho, ${ }^{1}$ and Stefano Zapperi ${ }^{2}$ \\ ${ }^{1}$ Departamento de Física, Universidade Federal do Ceará, 60451-970 Fortaleza, Ceará, Brazil \\ ${ }^{2}$ INFM UdR Roma 1 and SMC, Dipartimento di Fisica, Università "La Sapienza," P.le A. Moro 2, 00185 Roma, Italy
}

(Received 26 April 2002; revised manuscript received 17 July 2002; published 11 November 2002)

\begin{abstract}
We investigate flux penetration in a disordered type-II superconductor by molecular dynamics simulations of interacting vortices. We focus on the effect of different boundary conditions on the scaling laws for flux front propagation. The numerical results can be interpreted using a coarse-grained description of the system in terms of a nonlinear diffusion equation. We propose a phenomenological equation for the front position that captures the essential behavior of the system and recovers the scaling exponents.
\end{abstract}

DOI: 10.1103/PhysRevB.66.174507

PACS number(s): 74.60.Ge, 05.45.-a, 47.55.Mh

\section{INTRODUCTION}

In recent years, the discovery of high-temperature superconductors has generated a renewed interest in understanding the magnetization properties of type-II superconductors. ${ }^{1}$ The magnetization process is usually described in terms of the Bean model: ${ }^{2}$ as the magnetic flux enters into the sample from the boundaries, quenched disorder is responsible for the formation of a constant flux gradient. The Bean model provides a phenomenological picture of average magnetization properties, such as hysteresis and thermal relaxation, ${ }^{3}$ but does not describe local space-time fluctuations. Indeed, recent experiments showed that these fluctuations are not only common, but also large, spanning several length scales: the flux line dynamics is intermittent, taking place in avalanches, ${ }^{4}$ and flux fronts are fractal. ${ }^{5-7}$

A widely used modeling strategy to describe the fluctuations in the magnetization process consists in molecular dynamics (MD) simulations of interacting vortices, pinned by quenched random impurities. ${ }^{8-11}$ With this approach it has been possible to model flux profiles, ${ }^{9}$ hysteresis, ${ }^{9}$ avalanches, ${ }^{10,11}$ and plastic flow. ${ }^{8,11}$ One of the aims of these studies ${ }^{9}$ is to link the macroscopic behavior, as described, for instance, by generalized Bean models, to the microscopic vortex dynamics.

Recently, we have shown that the flux penetration due to interacting vortices in a disordered superconductor can be described by a disordered nonlinear diffusion equation. ${ }^{12}$ The equation can be obtained performing a coarse graining of the microscopic equation of motion of the vortices. In the absence of pinning, the equation reduces to the model introduced in Ref. 13. This model has been solved analytically to provide expressions for the dynamics of the front for different boundary conditions. ${ }^{13,14}$ When quenched disorder is included in the diffusion equations, flux fronts are pinned in agreement with MD simulations. Varying the parameters of the equation, we observe a crossover from flat to fractal flux fronts, consistent with experimental observations. The value of the fractal dimension suggests that the strong disorder limit is described by percolation. ${ }^{12}$ In the weak-disorder limit, we recover the analytical results derived in Refs. 13 and 14 . Using this description, we can thus directly link continuum theories, for which analytical solutions are possible, to the microscopic equations used in MD simulations. ${ }^{12}$
In this paper we systematically analyze the effect of different boundary conditions on the propagation of the flux front. A similar study was presented in Refs. 13 and 14 in the framework of nonlinear flux diffusion in the absence of disorder. We show here that the results are in agreement with MD simulations for various different boundary conditions. Next, we analyze the effect of disorder by varying the pinning strength in the MD system. The results are then interpreted theoretically by means of the nonlinear diffusion equation. ${ }^{12-14}$ Finally, we propose a phenomenological equation for the front position that is able to capture in a simple way the behavior of the system, recovering the numerical results for the different boundary conditions.

\section{VORTEX DYNAMICS MODEL}

In an infinitely long cylinder, flux lines can be represented as a set of interacting particles performing an overdamped motion in a random pinning landscape. ${ }^{8-11}$ The equation of motion for each flux line $i$ is given by

$$
\Gamma \vec{v}_{i}=\sum_{j} \vec{J}\left(\vec{r}_{i}-\vec{r}_{j}\right)+\sum_{p} \vec{G}\left[\left(\vec{R}_{p}-\vec{r}_{i}\right) / l\right],
$$

where the effective viscosity is obtained from material parameters as $\Gamma=\Phi_{0} H_{c 2} / \rho_{n} c^{2}$. Here, $\Phi_{0}$ is the magnetic quantum flux, $c$ is the speed of light, $\rho_{n}$ is the resistivity of the normal phase, and $H_{c 2}$ is the upper critical field. The first term on the right-hand side of Eq. (1) accounts for the vortex-vortex interaction and it is given by

$$
\vec{J}(\vec{r}) \equiv \Phi_{0}^{2} /\left(8 \pi \lambda^{3}\right) K_{1}(|\vec{r}| / \lambda) \hat{r},
$$

where $K_{1}$ is a Bessel function decaying exponentially for $|\vec{r}|>\lambda$, and $\lambda$ is the London penetration length. ${ }^{15}$ The interaction is cut off at a distance $6 \lambda$ to improve computational efficiency. The second term on the right-hand side of Eq. (1) describes the interaction between pinning centers, modeled as localized traps, and flux lines. Here, $\vec{G}$ is the force due to a pinning center located at $\vec{R}_{p}, l$ is the range of the wells (typically $l \ll \lambda$ ), and $p=1, \ldots, N_{p}\left(N_{p}\right.$ is the total number of pinning centers). For the pinning force, we use the following expression: $\vec{G}(\vec{x})=-f_{0} \vec{x}(|\vec{x}|-1)^{2}$, for $|\vec{x}|<1$ and zero 
otherwise. In the present simulations we restrict ourselves to the case $T=0$ (see Ref. 16 for the implementation of thermal noise in MD simulations).

As we discussed in the Introduction, we intend to study the effect of different boundary conditions on the flux penetration. We start with an empty system and concentrate all the vortices in a small strip at the boundary. Due to mutual repulsion, the vortices will be pushed inside the material, forming a flux front. There are several ways to implement the boundary conditions, corresponding to different experimental situations. Here, we will consider the following boundary conditions. ${ }^{13,14}$

(A) Constant total number of vortices. Experimentally this corresponds to an external control of the magnetic flux.

(B) Constant vortex concentration at the boundary. This case corresponds to an external control of the magnetic field.

(C) Total vortex number increasing at constant rate. This represents an external control of the flux rate.

(D) Boundary concentration increasing at constant rate, corresponding to a constant field rate.

As a word of caution, one should notice that boundary conditions can be more complicated in reality, due to complex surface barriers that oppose flux penetration. These are not considered here and the only surface barrier is provided by already entered flux lines.

\section{MD SIMULATIONS}

We perform MD simulations based on Eq. (1) and analyze the flux front propagation for different values of the pinning strength $f_{0}$. We typically use up to $N_{p}=800000$ Poissondistributed pinning centers of width $l=\lambda / 2$ in a system of size ( $\left.L_{x}=800 \lambda, L_{y}=100 \lambda\right)$, corresponding to a density of $n=10 / \lambda^{2}$. The number $N$ of flux lines depends essentially on the boundary condition adopted in the simulation. The injection of magnetic flux into the sample is implemented as in Ref. 12, concentrating at the beginning of the simulation all the flux lines in a small strip $L^{\prime} \ll \lambda$, parallel to the $y$ direction, and imposing periodic boundary conditions in both directions. The front position is taken as the $x$ coordinate of the most advanced particle in the system at different times.

Case (A), corresponding to a constant vortex number, was studied in detail in Ref. 12, where we showed that the front position $x_{p}$ grows initially with time as $t^{1 / 3}$ for small times. Eventually the front position slows down and saturates to a value $\xi_{p}$ which increases as the strength of the pinning centers $f_{0}$ is decreased. In particular, the front pinning length $\xi_{p}$ was found to scale as $f_{0}^{-1 / 2} \cdot{ }^{12}$ Here, we analyze the behavior of the front as a function of the pinning density. In Fig. 1 we report pinned density profiles for different values of $n$. We use a large density of pinning centers, corresponding to the weak-pinning regime. The data collapse indicates a scaling form of the type $\rho(x, n)=n^{1 / 4} \mathcal{G}\left(x n^{1 / 4}\right)$. This result combined with the one reported in Ref. 12 implies that the pinning length scales as $\xi_{p} \sim\left(f_{0} \sqrt{n}\right)^{-1 / 2}$. We notice that in the weakpinning regime one indeed expects the pinning strength to scale as $f_{0} \sqrt{n} .^{17}$

Next, we compare the behavior observed in case (A) with that of cases (B) $-(\mathrm{D})$. We first consider the case $f_{0}=0$, cor-

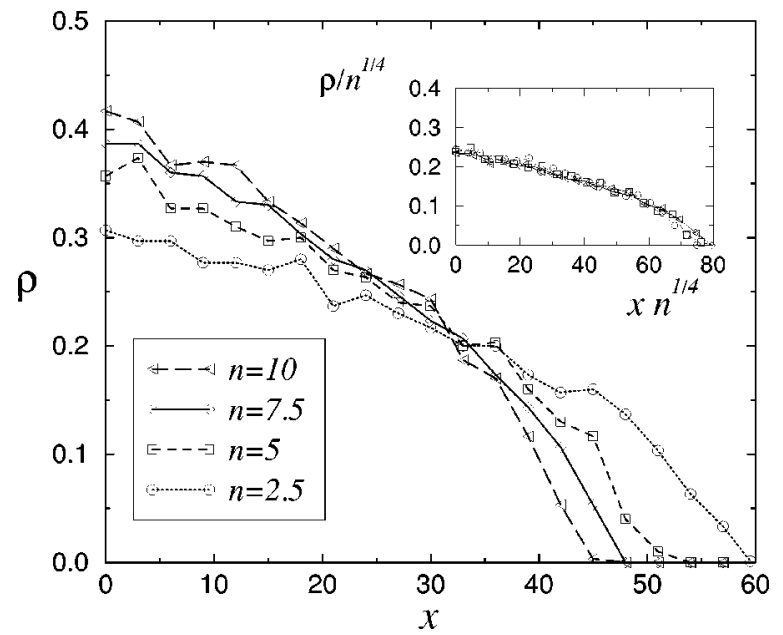

FIG. 1. The pinned density profiles measured in MD simulations for different values of the pinning center density. In the inset we show the data collapse.

responding to a clean superconductor (i.e., without defects) in order to clearly identify the front penetration law in the initial regime. In Fig. 2 we show that the front advances as a power law with an exponent that depends on the boundary condition. In particular, we find $x_{p} \sim t^{1 / 2}$ for case (B), $x_{p}$ $\sim t^{2 / 3}$ for case (C), and $x_{p} \sim t$ for case (D). The presence of disorder can affect all these behaviors in a different way, depending on the imposed boundary condition. Case (B) is quite similar to the case (A) studied in Ref. 12: after an initial transient, the front gets pinned and the pinning length scales with the pinning strength (see Fig. 3). In particular, the pinning length scales roughly as $x_{p} \sim 1 / f_{0}$, as shown in the inset of Fig. 3. For case (C), the front never gets pinned. The effect of disorder is only to slow down the dynamics (see Fig. 4). A similar behavior is found for case (D).

\section{NONLINEAR DIFFUSION}

In Ref. 12, we have shown that the front propagation can be described by coarse graining the system and obtaining a

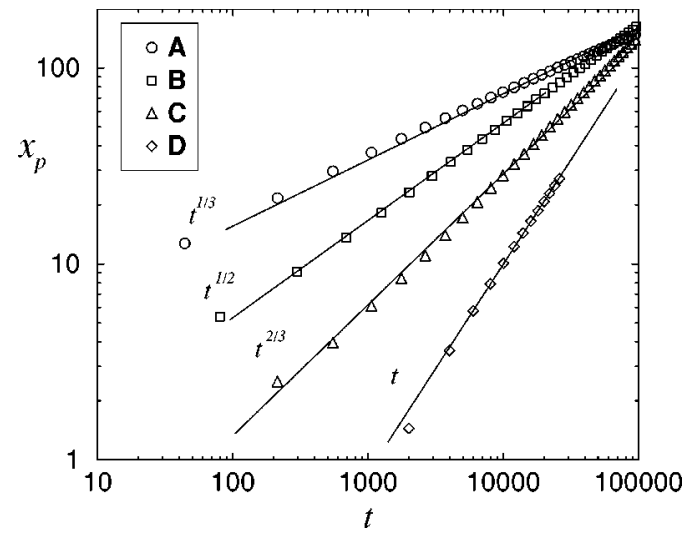

FIG. 2. The average position of the front plotted as a function of time. The data have been obtained from MD simulations with different boundary conditions (for a definition see text) in a clean system $\left(f_{0}=0\right)$. The curves increase as $t^{\alpha}$, where $\alpha$ depends on the particular boundary condition imposed. 


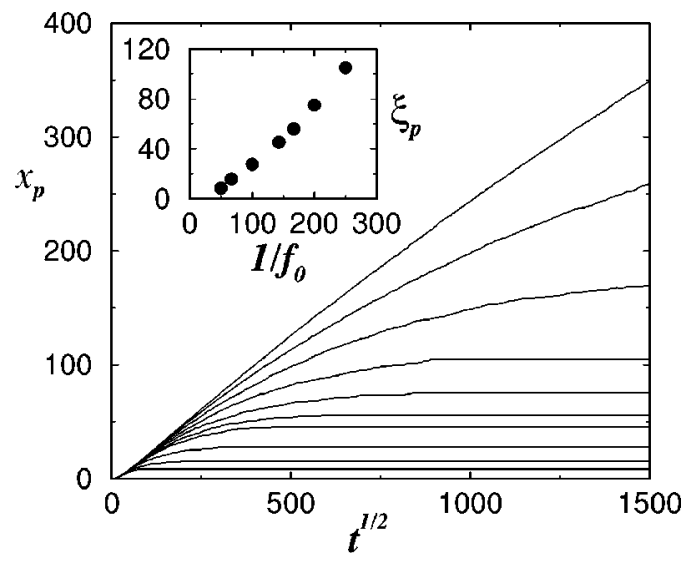

FIG. 3. The average position of the front, obtained from MD simulations for case (B), plotted as a function of time. The curves increase as $t^{1 / 2}$ and saturates at long times to a value depending on $f_{0}$. From top to bottom, the curves correspond to $f_{0}=0.001,0.002$, $0.003,0.004,0.005,0.006,0.007,0.01,0.015$, and 0.02. In the inset, we show that the pinning length scales as $f_{0}^{-1}$.

disordered nonlinear diffusion equation. The equation that rules the evolution of the local vortex density $\rho(\vec{r}, t)$ is

$$
\Gamma \frac{\partial \rho}{\partial t}=\vec{\nabla}\left(a \rho \vec{\nabla} \rho-\rho \vec{F}_{c}\right)+k_{B} T \nabla^{2} \rho,
$$

where $a \equiv \int d^{2} r \vec{r} \cdot \vec{J}(\vec{r}) / 2=\Phi_{0}^{2} / 4$ and $F_{c}$ is a random friction force due to the pinning centers, with a typical value scaling as $F_{c} \sim f_{0} \sqrt{n}$.

For $T=0$ and $f_{0}=0$, Eq. (3) can be solved exactly using scaling methods. ${ }^{13,14}$ In particular the density profiles obey the equation

$$
\rho(x, y, t)=t^{-\alpha} \mathcal{G}\left(x / t^{\beta}\right),
$$

where $\alpha$ and $\beta$ depend on the boundary conditions and satisfy $\alpha+2 \beta=1$. For the cases considered, (A) $\alpha=1 / 3, \beta$ $=1 / 3$; (B) $\alpha=0, \beta=1 / 2$; (C) $\alpha=-1 / 3, \beta=2 / 3$; and (D) $\alpha=-1, \beta=1$. These results are in perfect agreement with

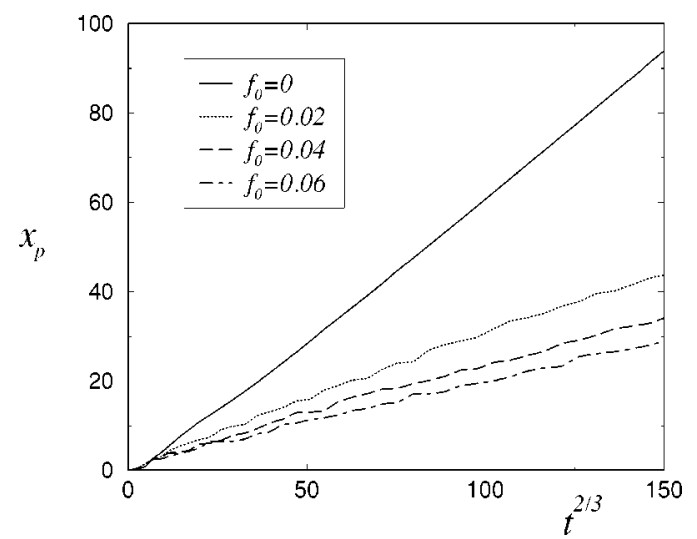

FIG. 4. The average position of the front for case (C) obtained from MD simulations for different values of the pinning strength. The front moves as $x_{p} \sim A t^{2 / 3}$, and $A$ is reduced in the presence of disorder.

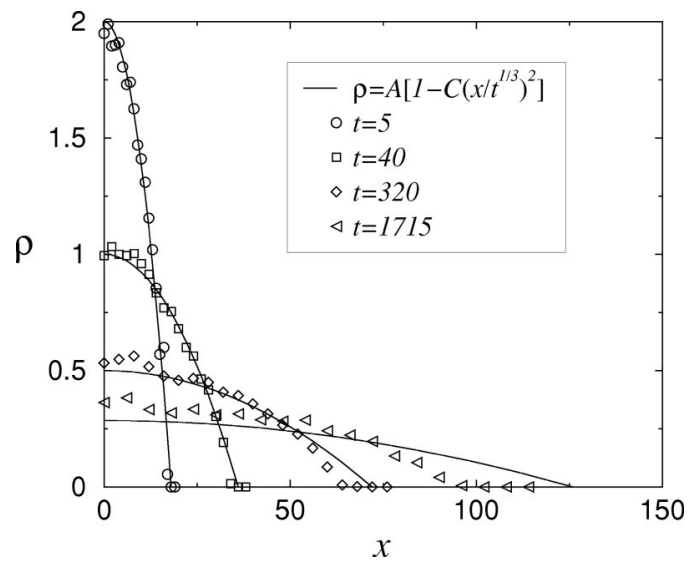

FIG. 5. The density profile at different times $t$ obtained from MD simulations for boundary condition (A) in the presence of disorder. At the beginning the profile follows the solution of the nonlinear diffusion equation in the disorder free case and eventually deforms due to the action of pinning.

the numerical simulations reported in Fig. 2, since the exponent $\beta$ describes the dynamics of the front position.

The function $\mathcal{G}(u)$ also depends on the boundary condition and for case (A) is given by $\mathcal{G}(u)=\left(1-u^{2}\right) / 6$ for $u$ $<1$ and vanishes for $u \geqslant 1$. The other cases are reported in Refs. 13 and 14 . We check by MD numerical simulations that, in the presence of disorder, the density profiles are described by the nonlinear diffusion equation (3). In Fig. 5 we show that the profiles follow the $f_{0}=0$ solution and then deform when pinning starts to dominate.

\section{FRONT DYNAMICS}

In order to understand in a simple way the effect of disorder on the front propagation for different boundary conditions, we can write an equation for the average position of the front. The approach is very similar in spirit to what is done for the imbibition of porous media. ${ }^{18}$ As discussed above, the front is driven by the density gradient against the pinning landscape. The density gradient can be estimated simply as $\nabla \rho \sim \rho(0, t) / x_{p}$, where $x_{p}$ is the front position and $\rho(0, t)$ is the boundary density. The typical pinning force can simply be taken as $f_{0} \sqrt{n}$. Collecting these two contribution we write

$$
\Gamma d x_{p} / d t=a \rho(0, t) / x_{p}-f_{0} \sqrt{n} .
$$

In order to close the problem we have to specify the behavior of the boundary density, which clearly will depend on the particular boundary condition chosen. Let us consider the various cases.

(A) When the total number of vortices is conserved, the boundary density decreases as the front advances. This can be explained by noting that the density at the boundary can be roughly estimated as $\rho(0, t)=m / x_{p}$, where $m \equiv M / L_{y}$ and $M$ is the the total number of vortices. Inserting this into Eq. (5) we obtain

$$
d x_{p} / d t=1 / x_{p}^{2}-g / m
$$


where $g \equiv f_{0} \sqrt{n} / a$ and time is expressed in units of $\Gamma /(m a)$. Equation (6) admits an implicit solution as

$$
(g / m) t=\operatorname{arctanh}\left(\sqrt{g / m} x_{p}\right) / \sqrt{g / m}-x_{p},
$$

which can be expanded for $x_{p} \ll 1 / \sqrt{g / m}$ to give $x_{p} \sim t^{1 / 3}$ (short times) and for $x_{p} \simeq 1 / \sqrt{g / m}$ (long times) yielding $x_{p}$ $\simeq \sqrt{m / g}\left\{1-2 \exp \left[-2(g / m)^{3 / 2} t\right]\right\}$, corresponding to a pinned front. This behavior is in agreement with the scaling found in numerical simulations (see Ref. 12).

(B) The case of a constant vortex density at the boundary is similar to case (A). The boundary condition is simply $\rho(0, t)=\rho_{0}$, and Eq. (5) becomes

$$
d x_{p} / d t=1 / x_{p}-g / \rho_{0},
$$

where time is now expressed in units of $\Gamma /\left(\rho_{0} a\right)$. As in the previous case, Eq. (8) cannot be solved explicitly but from the implicit solution it is possible to obtain the asymptotic behavior: $\quad x_{p} \sim t^{1 / 2}$ at short times and $x_{p} \simeq \rho_{0} /$ $g\left\{1-\exp \left[-\left(g / \rho_{0}\right) t\right]\right\}$ at long times, in agreement with the results presented in Fig. 3.

(C) This case is similar to case (A), with the difference that the total number of vortices increases with time (i.e., $m=h t$ ). Due to this, the front is never pinned. In the absence of pinning we recover the $t^{2 / 3}$ behavior observed in MD simulations. We cannot find an analytical solution of the equation in this case and resort to numerical integration. The results indicate that the front asymptotically grows as $x_{p}$ $=C+A t^{2 / 3}$. For low pinning we expect that the coefficient $A$ decreases with the pinning strength. This result is in agreement with the MD simulation (see Fig. 4).

(D) As in case (C), the front is not pinned by the disorder, which has the only effect of reducing the front velocity. One can compute the asymptotic velocity imposing $x_{p}=V t$ and inserting this expression in Eq. (5), which now reads

$$
(\Gamma / a) d x_{p} / d t=h t / x_{p}-g,
$$

where $h$ here is the rate of increase of the boundary density. Solving for $V$ one obtains $V=\left(\sqrt{g^{2}+4 h \Gamma / a}-g\right) a / 2 \Gamma$.

\section{DISCUSSION AND PERSPECTIVES}

In this paper we have analyzed the effect of different boundary conditions on the flux penetration in disordered type-II superconductors. We have conducted a series of MD simulations of interacting vortices and interpreted the results in terms of a nonlinear diffusion equation. In the limit of no disorder, the equation has been solved in Refs. 13 and 14, yielding solutions for the front propagation and the density profiles. Here we have shown that these results are in perfect agreement with MD simulations. Moreover, we have found that the presence of pinning centers affects the behavior of the system and, depending on the boundary conditions, the front is either pinned or simply slowed down. To clarify these effects, we have introduced a simple equation of motion for the front position, in the same spirit of the Washburn approach to imbibition. ${ }^{18}$ Despite its simplicity, the equation captures the essential features of the front dynamics.

We have focused our analysis in the weak collective pin-

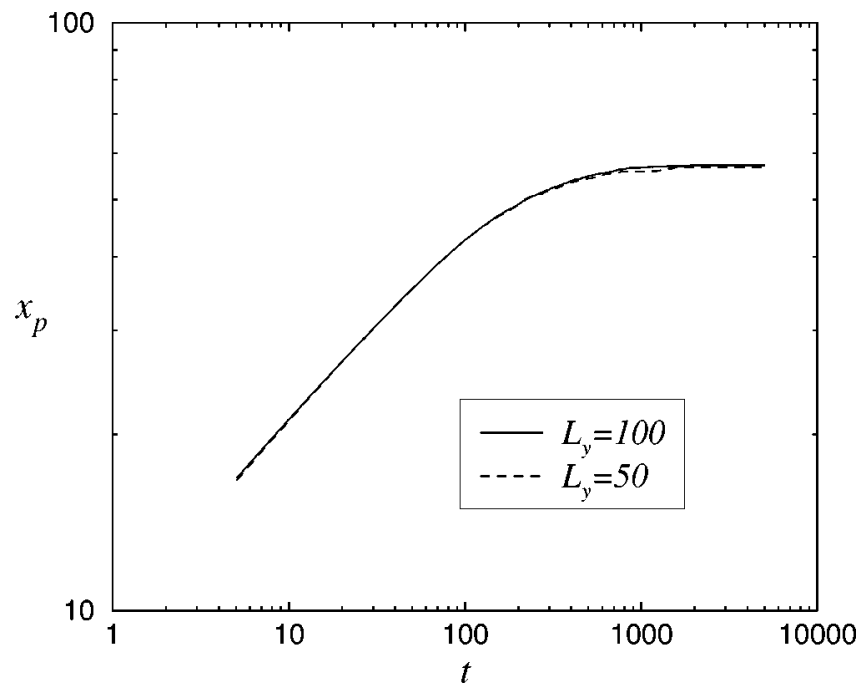

FIG. 6. The front position as a function of time computed from MD simulations for case (A) with two different system sizes $L_{y}$, keeping $m$ constant. The result does not depend on $L_{y}$.

ning regime, ${ }^{17}$ where we expect pinning to be due to the fluctuations in the pinning forces. Hence the front pinning length is controlled by $F_{c} \sim f_{0} \sqrt{n}$. Notice that in this regime the front, although fractal at small length scales, ${ }^{12}$ is well defined as in the experimental results presented in Refs. 5-7. This would not be the case in the strong-pinning regime where plastic flow is expected to be present and size effects become predominant. ${ }^{8}$ In our case, finite-size effects play no relevant role and the pinning length does not depend on the linear size of the lattice as can be seen in Fig. 6.

In our simulation we did not include the effect of the vortex core; thus we expect our results to be valid in the London regime for magnetic fields much smaller than $H_{c 2}$ so that vortices never overlap. In principle one could expect the result to be affected by the low distance singularity of the

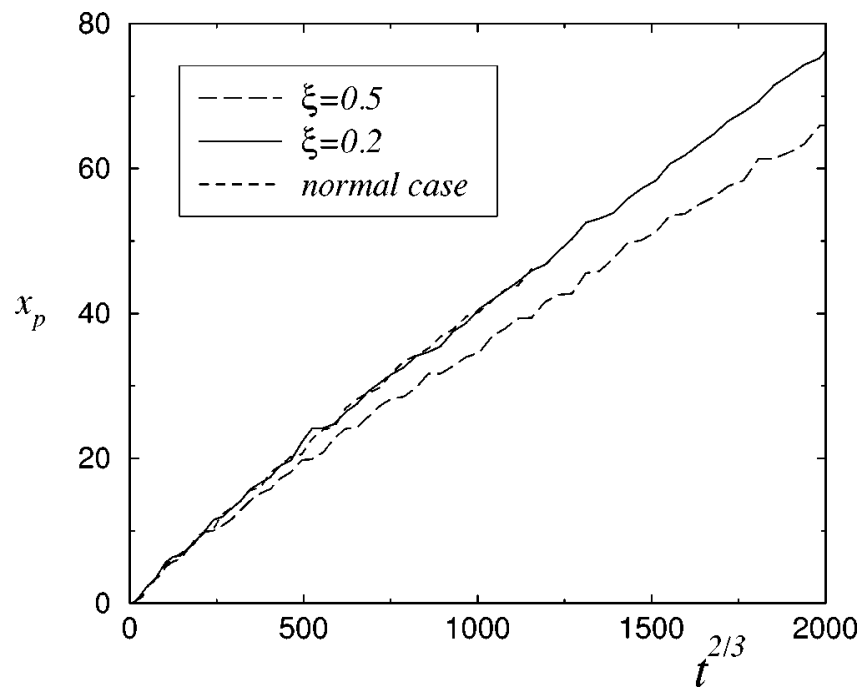

FIG. 7. The average position of the front for case (C) obtained from MD simulations including the cutoff at small length scale due to the vortex core. As in the case without cutoff, the front moves as $x_{p} \sim A t^{2 / 3}$, and $A$ is reduced for large $\xi$. 
vortex-vortex interaction we have adopted [see Eq. (2)]. A nonsingular expression for this interaction, taking explicitly into account the effect of the vortex cores, was proposed in Ref. 19:

$$
\vec{J}(\vec{r}) \equiv \Phi_{0}^{2} /\left(8 \pi \lambda^{3}\right)\left(K_{1}(|\vec{r}| / \lambda)-K_{1}(|\vec{r}| / \xi)\right) \hat{r} .
$$

In Ref. 20 it was shown that the low distance cutoff could generate vortex stacking (i.e., several vortices being captured by a single pin) at high vortex densities. We have tested the effect of vortex core on front propagation replacing the force in Eq. (2) with the one in Eq. (10) and studying case (C) for a pinning force of $f_{0}=0.02$ and $n=10$. The results in Fig. 7 show that the vortex core does not change substantially the dynamics of the front. We notice that the slope $A$ decreases as $\xi$ increases, which is due to the fact that the constant $a$ in Eq. (3) decreases as $\xi$ increases. Again the situation could change in the strong-pinning regime where vortex stacking becomes more important.

An important question that still remains to be addressed is the relevance of these results for experiments. We can associate each one of the boundary conditions studied here to different experimental conditions, corresponding to the way the field is applied to the sample. In many cases, the presence of surface barriers for flux penetration could in principle modify the scaling behavior discussed here. We believe, however, that away from the surface this effect should not be important and the scaling would be recovered. In addition, thermal and quantum creep effects in general may lead to a slowly moving front, even when pinning is expected. It is possible to account for these effects in this framework considering the diffusion term in Eq. (3) or adding a random noise term to the front propagation equation. In particular conditions, the time scale of these creep processes could be large enough to allow for the observation of front pinning.

In conclusion, we expect that our approach together with a systematic series of magneto-optical measurements performed under different magnetic field controls (e.g., see Refs. 5-7), will represent significant steps for the understanding of the flux penetration phenomenon in disordered superconductors. Our results could also be generalized to interpret magnetization curves or hysteresis loops as a function of the field driving frequency.

\section{ACKNOWLEDGMENTS}

This work has been supported by CNPq and FUNCAP. S.Z. is grateful for the hospitality of the Physics Department of UFC where this work has been completed.
${ }^{1}$ G. Blatter, M.V. Feigel'man, V.B. Geshkenbein, A.I. Larkin, and V.M. Vinokur, Rev. Mod. Phys. 66, 1125 (1994).

${ }^{2}$ C.P. Bean, Rev. Mod. Phys. 36, 31 (1964).

${ }^{3}$ Y.B. Kim, C.F. Hempstead, and A.R. Strnad, Phys. Rev. 129, 528 (1963)

${ }^{4}$ S. Field, J. Witt, F. Nori, and X. Ling, Phys. Rev. Lett. 74, 1206 (1995); C.M. Aegenter, Phys. Rev. E 58, 1438 (1998); K. Behnia, C. Capan, D. Mailly, and B. Etienne, Phys. Rev. B 61, R3815 (2000).

${ }^{5}$ R. Surdeanu, R.J. Wijngaarden, E. Visser, J.M. Huijbregtse, J.H. Rector, B. Dam, and R. Griessen, Phys. Rev. Lett. 83, 2054 (1999).

${ }^{6}$ R. Surdeanu, R.J. Wijngaarden, B. Dam, J. Rector, R. Griessen, C. Rossel, Z.F. Ren, and J.H. Wang, Phys. Rev. B 58, 12467 (1998)

${ }^{7}$ S.S. James, S.B. Field, J. Seigel, and H. Shtrikman, Physica C 332, 445 (2000).

${ }^{8}$ H.J. Jensen, A. Brass, and A.J. Berlinsky, Phys. Rev. Lett. 60, 1676 (1988).

${ }^{9}$ C. Reichhardt, J. Groth, C.J. Olson, S. Field, and F. Nori, Phys.
Rev. B 52, 10441 (1995); 53, R8898 (1996).

${ }^{10}$ O. Pla, N.K. Wilkin, and H.J. Jensen, Europhys. Lett. 33, 297 (1996).

${ }^{11}$ C.J. Olson, C. Reichhardt, and F. Nori, Phys. Rev. B 56, 6175 (1997); Phys. Rev. Lett. 80, 2197 (1998).

${ }^{12}$ S. Zapperi, A.A. Moreira, and J.S. Andrade, Jr., Phys. Rev. Lett. 86, 3622 (2001).

${ }^{13}$ V.V. Bryskin and S.N. Dorogotsev, JETP 77, 791 (1993); Physica C 215, 173 (1993).

${ }^{14}$ J. Gilchrist and C.J. van der Beek, Physica C 231, 147 (1994).

${ }^{15}$ P.-G. de Gennes, Superconductivity of Metals and Alloys (Benjamin, New York, 1966).

${ }^{16}$ D. Monier and L. Fruchter, Eur. Phys. J. B 17, 201 (2000).

${ }^{17}$ A.I. Larkin and Yu.N. Ovchinnikov, J. Low Temp. Phys. 34, 409 (1979).

${ }^{18}$ E. Washburn, Phys. Rev. 17, 273 (1921). For a review see M. Dube, M. Rost, and M. Alava, Eur. Phys. J. B 15, 691 (2000).

${ }^{19}$ E.H. Brandt, Phys. Rev. B 34, 6514 (1986).

${ }^{20}$ D.K. Jackson et al., Europhys. Lett. 52, 210 (2000). 